\title{
Effect of the perinatal risk factors on the outcome of the term asphyxiated neonates at the time of discharge in NICU
}

\author{
Sahoo $\mathbf{M R}^{1}$, Arigela $\mathbf{V}^{2}$, Pradeep $\mathbf{Y}^{3}$, Rao $\mathbf{S}^{4}$, Sudarsini $\mathbf{P}^{5}$. \\ ${ }^{1}$ Dr Manas Ranjan Sahoo, FNB (PIC), Assistant Professor, Department of Pediatrics, ${ }^{2}$ Dr Vasundhara Arigela, Pediatrics, \\ Professor, Department of Pediatrics, ${ }^{3}$ Dr Y Pradeep, Post Graduate, Department of Pediatrics, ${ }^{4}$ Dr Srinivasa Rao, \\ Professor, Department of Pediatrics, ${ }^{5}$ Dr P Sudarsini, Professor \& HOD, Department of Pediatrics. All are affiliated with \\ ASRAM Medical College, Eluru, AP, India.
}

Address for Correspondence: Dr Manas Ranjan Sahoo, FNB (PIC), Assistant Professor, Department of Pediatrics, ASRAM Medical College, Eluru, AP. Mail id: drmrsahoo@gmail.com

\begin{abstract}
Hypoxic-ischemic encephalopathy (HIE) secondary to perinatal asphyxia remains a major cause of neonatal mortality and morbidity worldwide. Perinatal asphyxia was responsible for $20 \%$ of all neonatal deaths. Manifestations of HIE were seen in approximately $1.5 \%$ of all babies. Aims and Objectives of the study: 1.To study the various perinatal risk factors which are contributing to HIE. 2. To study the outcome of the term asphyxiated neonates at the time of discharge in relation to the perinatal risk factors. Materials and method: This prospective study was conducted on the term asphyxiated babies who were admitted in ASRAM medical college, Eluru during the period of January 2013 to August 2014. Result: The incidence of the HIE in neonates in the present study is $15.5 \%$. Males neonates were more in number $40(61.5 \%)$ than female neonates $25(38.5 \%)$. Cord entangled twice around the neck was having very poor prognosis 83.4\%. Conclusion: In the present study cord around the neck, the neonates having Apgar score $<3$ at 5 min have shown poor prognosis and deaths. Pediatrician presence at the time of delivery is associated with good prognosis in the neonates.
\end{abstract}

Key words: Hypoxic-ischemic encephalopathy, APGAR Score, Neonatal Resuscitation

\section{Introduction}

Hypoxic-ischemic encephalopathy (HIE) secondary to perinatal asphyxia remains a major cause of neonatal mortality and morbidity worldwide. The definition of perinatal asphyxia is variably indistinct. "Failure to initiate or sustain respiration after birth" has been defined as criteria for the diagnosis of asphyxia by WHO [1].

All the following must be present for designation of asphyxia [2].

(a) Profound metabolic or mixed acidemia $(\mathrm{pH}<7.00)$ in cord blood.

(b) Persistence of Apgar scores 0-3 for longer than 5 minutes.

(c) Neonatal neurologic sequelae (eg, seizures, coma, Hypotonia).

Manuscript received: $28^{\text {th }}$ March 2016 Reviewed: $14^{\text {th }}$ April 2016

Author Corrected; $26^{\text {th }}$ April 2016

Accepted for Publication: $12^{\text {th }}$ May 2016 (d) Multiple organ involvement (eg, kidney, lungs, liver, heart, intestine)

Definitions based on Apgar scores may be useful as it can be used for formulating guidelines for postasphyxial treatment of neonates. Apgar scores are also useful for predicting long term outcome in infants with perinatal asphyxia $[3,4]$.

Hypoxic-ischemic cerebral injury results from a combination of hypoxemia and ischemia, which often is associated with impaired cerebrovascular auto regulation and exacerbated by diminished cerebral glucose substrates, lactic acidosis, the accumulation of free radicals and excitotoxic aminoacids (especially glutamate) and other metabolic derangements. The localization and the extent of the perinatal Hypoxic Ischemic cerebral injury is determined principally by the maturity of the brain at the time of the insult. 
Indian data: According to NNPD 2003 data collected from 17 tertiary neonatal intensive care units in India, Apgar scores <7 at 1 minute (includes moderate and severe asphyxia) were documented in $9 \%$ of all intramural deliveries [1]. 22.5\% babies continued to have Apgar scores $<7$ at 5 minutes of age. Perinatal asphyxia was responsible for $20 \%$ of all neonatal deaths. Manifestations of HIE were seen in approximately $1.5 \%$ of all babies. Perinatal asphyxia was the commonest cause of still-births accounting for one-third of all such cases. Perinatal asphyxia is the most important cause of preventable cerebral injury occurring in the neonatal period. Full recovery may not occur and many children are left with lifelong neurological impairment and in some cases, incapacitating disability. Data from National Neonatal Perinatal database suggests that perinatal asphyxia contributes to almost $20 \%$ of neonatal deaths in India. Between $20 \%$ and $50 \%$ of newborn infants affected by perinatal brain injury die during the newborn period, and $25-60 \%$ of the survivors suffer from permanent neurodevelopmental handicaps, including cerebral palsy, seizures, mental retardation, and learning disabilities [5-7]. This creates a great burden for the family, as well as for the society. Seizures occur in approximately $50 \%$ of asphyxiated infants are indicative of moderate or severe encephalopathy.

\section{Aims and objectives of the study}

1. To study the various perinatal risk factors which are contributing to HIE.

2. To study the outcome of the term asphyxiated neonates at the time of discharge in relation to the perinatal risk factors.

\section{Materials and Methods}

This prospective study was conducted on the term asphyxiated babies who were admitted in NICU of ASRAM medical college, Eluru, AP, India during the period of January 2013 to August 2014 .

Study design: A Descriptive retrospective history was taken for all the term babies with perinatal asphyxia admitted from January 2013 to August 2014 at the neonatal ICU of ASRAM medical college, Eluru. Total number of cases 65 , were included in the study using the following inclusion and exclusion criteria.

\section{Inclusion criteria:}

-All the term babies with the history of perinatal asphyxia who were admitted to ASRAM hospital, including inborn and outborn neonates.

-The study includes full term newborns having a clinical history consistent with either of the following.

1. Perinatal asphyxia by 5 minute APGAR score < 5 [ 8]

2. Failure to initiate or sustain respiration after birth [1]

3. Clinical post asphyxial syndrome (encephalopathy, seizures).

\section{Exclusion criteria :}

- Babies who were born with congenital anomalies, progressive metabolic diseases, brain malformations, acquired central nervous system infections [9].

- Babies who have left the hospital without continuing the treatment completely.

- Babies having low APGAR scores for other reasons like as follows: - Birth weight $<1500 \mathrm{gms}$, prematurity, cyanotic congenital heart diseases, congenital infections were excluded.

Consent: Informed written consent will be obtained from a parent after a full verbal and written explanation of the study. The attending physician will explain parents during the intervention period to ensure that they understand the study procedures and continue to consent to participate in the study. After consent and enrollment their detailed history including peri-natal history, APGAR score, resuscitation measures, problems and outcome were recorded through a structured questionnarie on a pre-designed study proforma. Clinical examination of all the admitted term asphyxiated babies were done. The evaluation was done including the general examination such as anthropometry, signs and stages of hypoxic ischemic encephalopathy (HIE) as proposed by sarnat and sarnat staging were assessed. According to this classification.

1. HIE stage 1 (Mild) includes irritability, hyperalertness, normal tone, strong moro's reflex, mydriasis.

2. HIE stage 2 (Moderate) includes lethargy, seizures, marked abnormalities of tone and requirement of tube feeding.

3. HIE stage 3 (Severe), comatose, prolonged seizures, severe hypotonia and failure to maintain spontaneous respiration.

The early outcome were recorded after 72 hours of admission it was measured as clinical improvement, death and/ presence of HIE stage I, II, III. At the time of the discharge the outcome has been assessed by seizure control, feeding pattern, and clinical neurological evaluation. Babies were been divided into good and poor outcomes based on the following criteria. 
Good- Those babies with no feeding difficulties and taking breast feeds, having seizures controlled well or baby being on single AED at the time of discharge and neurologically normal clinical assessment.

Poor- those babies with feeding difficulties like baby on spoon /tube feeds, having refractory seizures or seizures controlled with 2 or more AED's at the time of discharge and abnormal neurological clinical assessment.
Ethical issuses and stastiscal analysis methods: Approval for the study has been obtained from the ASRAM medical college Ethics Committee. Ethics committee clearance certificate has been issued.

All the data were been taken from the history and the consent has been taken from the parents. The complete data has been kept in the form of a master sheet in the Microsoft word excel 2013 format and the statistical data tables were proceeded using the SPSS 17.0 version software.

\section{Results}

Totally 451 neonates were admitted in the NICU of ASRAM medical college during the study period in which 70 $(15.5 \%)$ neonates were noted to be term asphyxiated newborns. Males were more in number in the study showing that 40 $(61.5 \%)$ and female neonates $25(38.5 \%)$ in number. Both the sexes have an almost equal percentage of deaths and the prognostication with $7.5 \%$ deaths in male babies and $8 \%$ deaths in female neonates. HIE neonates were 65 in number, in which $53.84 \%$ have good prognosis, $38.46 \%$ poor prognosis and $7.69 \%$ died. Neonates born through NVD or through LSCS have an almost equal prognostication showing the death percentages as $7.5 \%$ in LSCS deliveries and $7.9 \%$ in normal deliveries, 55\% babies born through NVD have good prognosis, and $36.8 \%$ have poor prognosis. $51 \%$ babies born through LSCS deliveries have good prognosis and $40.7 \%$ have poor prognosis. Neonates born with meconium staining or without meconium staining have an almost equal prognostication showing an insignificant $\mathrm{p}$ value. Death percentages were all most equal in both with $8 \%$ and $7.5 \%$. Neonates born with meconium staining have $40 \%$ of poor prognosis and $52 \%$ good prognosis. Neonates without meconium staining have $37.5 \%$ poor prognosis and $55 \%$ good prognosis.

Table 1: Relation of the maternal gravida to prognosis of the neonates.

\begin{tabular}{|l|l|l|l|l|l|}
\hline \multirow{3}{*}{ Prognosis } & \multicolumn{5}{|c|}{ Gravida } \\
\cline { 2 - 6 } & Primigravida & Multigravida & Total \\
\cline { 2 - 7 } & No & $\%$ & No. & \% & No \\
\hline Good & 27 & $50.9 \%$ & 8 & $66.7 \%$ & 35 \\
\hline Poor & 21 & $39.6 \%$ & 4 & $33.3 \%$ & 25 \\
\hline Death & 5 & $9.4 \%$ & 0 & $0 \%$ & 5 \\
\hline Total & 53 & & 12 & \multicolumn{2}{|l}{6} \\
\hline
\end{tabular}

$50.9 \%$ neonates born to primigravida mothers have good prognosis and the remaining $39.6 \%$ have poor prognosis, and $9.4 \%$ of neonates died. $67 \%$ neonates born to multigravida mothers have good prognosis and $33 \%$ have poor prognosis and no deaths occurred.

Table 2: Relation of the cord around the neck to prognosis of the neonates.

\begin{tabular}{|c|c|c|c|c|c|c|c|}
\hline \multirow[t]{3}{*}{ Prognosis } & \multicolumn{7}{|c|}{ Cord around the neck of the neonates } \\
\hline & \multicolumn{2}{|c|}{ Yes (twice) } & \multicolumn{2}{|c|}{ Yes (once) } & \multicolumn{2}{|l|}{ No } & \multirow{2}{*}{$\begin{array}{l}\text { Total } \\
\text { No }\end{array}$} \\
\hline & No & $\%$ & No & $\%$ & No & $\%$ & \\
\hline Good & 0 & $0 \%$ & 1 & $25 \%$ & 34 & $61.8 \%$ & 35 \\
\hline Poor & 5 & $83.4 \%$ & 3 & $75 \%$ & 17 & $31 \%$ & 25 \\
\hline Death & 1 & $16.6 \%$ & 0 & $0 \%$ & 4 & $7.2 \%$ & 5 \\
\hline Total & 6 & & 4 & & 55 & & 65 \\
\hline
\end{tabular}

Pearson Chi-Square Tests: Chi-square Value is 11.287 for $4 \mathrm{~d}$. f at 5\% L.O.S and P - Value is 0.024 (Significant) Out of all neonates with cord entangled twice around the neck $83.4 \%$ have poor prognosis and death have been noticed in $16.6 \%$ cases. Out of neonates with cord entangled once around the neck $75 \%$ have poor prognosis, $25 \%$ have good prognosis. 
Out of all neonates without any cord around the neck $31 \%$ have poor prognosis, $61.8 \%$ have good prognosis and $7.2 \%$ cases died. The 4 neonates which were having no cord entanglement, and having the Apgar score $<3$ at 10 mins have expired within 24 hours of life after admission.

Table 3: Relation of APGAR score timing to the prognosis of the neonates.

\begin{tabular}{|l|l|l|l|l|l|l|l|}
\hline \multirow{2}{*}{ Prognosis } & \multicolumn{6}{l}{ APGAR Scoring at 5minutes } \\
\cline { 2 - 8 } & $<\mathbf{3}$ & $.0 \%$ & 0 & $.0 \%$ & 35 & $100.0 \%$ & 35 \\
\hline Good & 0 & $.0 \%$ & 25 & $100.0 \%$ & 0 & $.0 \%$ & 25 \\
\hline Poor & 0 & $100.0 \%$ & 0 & $.0 \%$ & 0 & $.0 \%$ & 5 \\
\hline Death & 5 & & 25 & & 35 & & 65 \\
\hline Total & 5 & & & & & \\
\hline
\end{tabular}

Pearson Chi-Square Tests: Chi-square Value is 130.00 for $4 \mathrm{~d}$. f at 5\% L.O.S and P- Value is 0.000 ( Highly Significant).

APGAR scoring has a very good value in the study showing as a good indicator for the prognosis of the neonate. The neonates born with APGAR score $<3$ at 5 mins have 100\% deaths and the neonates born with the APGAR score between 3 to 5 at $5 \mathrm{mins}$ have an $100 \%$ poor prognosis and the neonates born with APGAR score > 5at $5 \mathrm{mins}$ have $100 \%$ good prognosis.

The neonates included under stage 1 have $94.5 \%$ good prognosis and 5.5\% poor prognosis. Neonates included in the stage 2 were having an equal percentages $47.4 \%$ good and poor prognosis and death percentage of $5.2 \%$. On a overall view, $33.3 \%$ babies with stage 3 died and $66.7 \%$ have poor prognosis.

The neonates in which the AED's were continued at the time of discharge, $59.5 \%$ have poor prognosis and $40.5 \%$ have good prognosis. And in the neonates where the AED's were not continued, 100\% have good prognosis.

The ventilation requirement in the HIE neonates with a central cause has shown that $54.6 \%$ neonates have a poor prognosis and $22.7 \%$ with death and the remaining $22.7 \%$ with a good prognosis. The neonates who do not require ventilator support have $69.7 \%$ of good prognosis and only $30.3 \%$ of poor prognosis and no deaths noticed.

Table 4: Relation with presence of Pediatrician to deaths due to asphyxia in neonates.

\begin{tabular}{|l|l|l|l|l|l|}
\hline Prognosis & \multicolumn{4}{l|}{ Pediatric Assistance } & \multicolumn{2}{l|}{ Total } \\
\hline & Yes & No & $\%$ & No \\
\hline & No & $\%$ & No & $80 \%$ & 5 \\
\hline Death & 1 & $20 \%$ & 4 & & 5 \\
\hline
\end{tabular}

Pearson Chi-Square Tests: Chi-square Value is 6.476 for 2 d.f at 5\% L.O.S and P - Value is 0.039 (Significant). When there is a pediatrician at the time of delivery only $2.6 \%$ of deaths were noticed but when a pediatrician is not available then death percentage was $15.5 \%$.

\section{Discussion}

No exact statistics are available on perinatal asphyxia and its complications in India. In our study, $15.5 \%$ of infants at our neonatal service were diagnosed with asphyxia during the 1year period. Similar results have been observed in other study [10]. Our result concluded gender was not a risk factor to affect the prognosis in asphyxiated newborn. Primigravida was a risk factor for poor prognosis as described in a study by a study in
Saudi Arabia [11]. Mode of delivery did not affect the outcome. Another study performed by Agarwal et al shows that modes of delivery did not affect the progression to different stages of HIE in neonates with perinatal asphyxia [12]. Cord around the neck was a definite risk factor for poor prognosis. Twice around the neck was also responsible for more no of deaths in compared to no cord around the neck. Even though cord around the neck are common and occur $25 \%-35 \%$ of 
the time rarely a neonates cord will be wrapped so tightly around their neck that their body is compressed and oxygen delivery to the neonate is compromised. When this occurs, a caesarean is prudent and in its absence, perinatal asphyxia/death could occur. Cords around the neck mostly are of single and are loosely coiled and less commonly they are tightly coiled. Mercer et al study shows that cord around the neck of $10 \%-37 \%$ of births, shown to be having short term morbidity. Although studies have shown a clear evidence that not cutting a tight cord around the neck before, or immediately after birth can result in serious brain injuries and even death $[13,14,15]$.

Meconium staining does not affect the outcome. Similar results also have been described by various studies $[16,12]$.

The present study describes that any neonates born with an APGAR score of $<3$ at $5 \mathrm{~min}$ have $100 \%$ mortality occurrence and if APGAR score of 3- 5 have $100 \%$ poor prognosis. The neonate with APGAR score of $>5$ have $100 \%$ good prognosis. Similarly, Nagdyman et al. found that one- and five-minute APGAR scores of patients with asphyxia were statistically significantly lower than those of control group [17]. The APGAR scoring system has been established as an important predictor of prognosis in asphyxia cases.

It was coincident with a high neonatal encephalopathy grade, low Apgar scores at 5 minutes, early neonatal seizures, and necessity of antiepileptic drug therapy. These clinical variables maintain a close relationship with the cases in this sample with unfavorable outcomes and hypoxic-ischemia as the etiologic factor.

All the HIE neonates in the present study are staged into 4 parameters based on Sarnat and sarnat staging clinically, than those staged neonates are related with the prognosis of the neonates. The neonates with stage I have good prognosis and the neonates which were staged under stage 2 and stage 3 have a higher incidence of poor prognosis and deaths.

Ventilator requirement has been a poor prognostic factor in outcome of HIE. Mortality as well as morbidity has been seen more in ventilated babies. Some studies have reported similar results [18].

The number of AED's used in the neonates for controlling the seizures also decide the prognosis of the neonates. The neonates with the early usage of AED's have a good prognosis. Usage of multiple AED's in neonates have poor prognosis. Usage of three AED's in a neonates with controlled seizure has higher mortality and moribidity. The neonates in which an AED is not used and when there is no clinical appearance of seizures those neonates have good prognosis.

The present study shows that how important a pediatrician presence for a neonatal resuscitation, showing a varied change in prognosis of the neonate. The death percentage in the absence of the pediatrician for a neonatal resuscitation is $80 \%$ and in the presence of pediatrician it is only $20 \%$.

A study conducted in Thailand shows that the presence of a Paediatrician during the neonatal resuscitation has shown the importance in prognosis of neonates as in our study [10].

\section{Conclusions}

In conclusion, we can take necessary preventive measures to reduce incidence of perinatal asphyxia. We think, paediatrician presence at the time of the neonatal resuscitation will improve the outcome. Low APGAR score and cord around neck are two bad prognostic factors in the outcome of perinatal asphyxia.

Funding: Nil, Conflict of interest: Nil

Permission from IRB: Yes

\section{References}

1. World Health Organizaton. Perinatal mortality: a listing of available information. FRH/MSM.96.7. Geneva:WHO,1996.

2. ACOG Committee opinion.Use and abuse of the Apgar score.Number 174-July 1996. (replaces No 49, November 1986). Committee on Obstetric practice and American Academy of Pediatrics: Committee on Fetus and Newborn. American College of Obstetricians and Gynecologists.Int JGynaecol Obstet,1996.54(3):p303-5.

3. Casey BM, McIntire DD, Leveno KJ. The continuing value of the Apgar score for the assessment of newborn infants. New Engl J Med 2001;344(7):467-71

4. Moster D, Lie RT, Irgens LM, Bjerkedal T, Markestad T. The association of Apgar score with subsequent death and cerebral palsy: A population based study in term infants. J Pediatr 2001;138(6):798803. 
5. Azzopardi DV, Strohm B, Edwards AD, Dyet L, Halliday HL, Juszczak E, Kapellou O, Levene M, Marlow N, Porter E, Thoresen M, Whitelaw A, Brocklehurst P, TOBY Study Group: TOBY Study Group. Moderate hypothermia to treat perinatal asphyxial encephalopathy. N Engl J Med 2009, 361(14):1349-1358.

6. Jacobs SE, Morley CJ, Inder TE, Stewart MJ, Smith KR, McNamara PJ, Wright IM, Kirpalani HM, Darlow BA, Doyle LW, Infant Cooling Evaluation Collaboration: Infant Cooling Evaluation Collaboration. Whole-bodyhypothermia for term and nearterm newborns with hypoxic ischemic encephalopathy: a randomized controlled trial. Arch Pediatr Adolesc Med 2011, 165(8):692-700.

7. Simbruner G, Mittal RA, Rohlmann F, Muche R: neo.nEURO.network trial participants. Systemic hypothermia after neonatal encephalopathy: outcomes of neo-.nEURO.network RCT. Pediatrics 2010, 126(4): e771-e778.

8. Memon, Shazia; Shaikh, Salma; Bibi, Seema . To compare the outcome (early) of neonates with birth asphyxia in-relation to place of delivery and age at time of admission. Journal of the Pakistan Medical Association; 2013, 62 (12), p1277.

9. Luis Fernando Garcias da Silva, João Rubião Höefel Filho, Maurício Anés, and Magda Lahorgue Nunes, Prognostic Value of 1H-MRS in Neonatal Encephalopathy Pediatric Neurology 2006, 34 (5 ): 360 $-366$.

10. Futrakul S, Praisuwanna P, Thaitumyanon P . Risk factors for hypoxic- ischemic encephalopathy in asphyxiated newborn infants. Journal of theMedical Association of Thailand $=$ Chotmaihet Thangphaet 2006, 89(3):322-328.

11. Itoo BA et al. Hypoxic ischemic encephalopathy. Incidence and risk factors in North Western Saudi Arabia.Saudi Med J 2003,24(2):147-53.

12. Agrawal et al . Electrocardiographic and enzymatic correlations with outcome in neonates with hypoxicischemic encephalopathy.Italian journal of pediatrics 2012, 38:33 .p.1-5.

13. Jefford.E., Fahyk., et al. (2009). routine vaginal examinationto check for a nuchal cord Br J Midwifery, 2009, 17(4):246-49.

14. Mercer et al.(2005)" Nuchal cord management and Nurse- Midwifery practice", Journal of Midwifery and women's health 2005,50(5):373-379.

15. Iffy L.,Varadi V. and Papp E.(2001).'Untoward neonatal sequelae deriving from cutting of the umbilical cord before delivery”. Medlaw 2001, 20(4):627-34.

16. Martin-Ancel et al.Multiple organ involvement in perinatal asphyxia. J Pediatr 1995, 127(5):786-93.

17. Nagdyman N, Grimmer I, Scholz T, et al. Predictive value of brain-specific proteins in serum for neurodevelopmental outcome after birth asphyxia. Pediatr Res. 2003,54(2):270-5.

18. Gül A, Cömert S, Ağzıkuru T. Retrospective Analysis of the cases of perinatal asphyxia. J Kartal TR 2010;21(2):77-83.

\section{How to cite this article?}

Sahoo MR, Arigela V, Pradeep Y, Rao S, P Sudarsini P. Effect of the perinatal risk factors on the outcome of the term asphyxiated neonates at the time of discharge in NICU. Int J Pediatr Res 2016;3(5):340345.doi:10.17511/ijpr.2016.i05.12 